\title{
COMPETENCIAS DEL DOCENTE-TUTOR. DIÁLOGO Y ACOMPAÑAMIENTO EN EL AULA
}

Concepción Barrón Tirado*

\section{Resumen}

Hoy en día la educación superior debe enfrentar retos particularmente difíciles como el de formar profesionales capaces de generar y conducir los cambios de la sociedad, además de incidir de manera cada vez más decidida, permanente y eficaz en sus ámbitos. Todo ello trae consigo un amplio debate sobre el futuro de la Educación Superior y genera propuestas que marcan una visión distinta.

En sentido amplio:

"La misión de la educación para la era planetaria es fortalecer las condiciones de posibilidad de la emergencia de una sociedad-mundo compuesta por los ciudadanos protagonistas, consciente y críticamente comprometidos en la construcción de una civilización planetaria” (Morín, 2003, p. 122).

Entre los temas abordados dentro de los debates destaca el de los sistemas educativos y la necesidad de revisarlos y transformarlos para enfrentar demandas de una nueva naturaleza, asociadas a un mundo globalizado en el que se encuentran insertas las sociedades.

\section{Abstract}

Nowadays Higher Education, must face particularly difficult challenges like training professionals able to generate and lead the changes of society, besides affecting in a more decided, permanent and effective way in its scopes. This brings an ample debate on the future of Higher Education and generates proposals that mark a different vision.

Broadly speaking:

* Investigadora de Carrera Titular "B", de Tiempo Completo Definitiva .IISUEUNAM, Coordinadora del programa de posgrado en pedagogía de la UNAM, Miembro del Sistema Nacional de Investigadores Nivel I, Doctora en Pedagogía, con Mención Honorífica. UNAM, Asistencia y participación en diversos Eventos Académicos a nivel nacional e internacional. Coordinadora de diversos curso en el área de formación de docentes. Docente de la licenciatura en Pedagogía en La Facultad de Filosofía y Letras de la UNAM, docente del Posgrado UAT y UAEM. Líneas de Investigación: Didáctica, Curriculum y Trabajo. Participación en los estados del conocimiento: Seguimiento de egresados en el área de Educación 
Economía y Educación y en el de Currículo, coordinado por el COMIE. Publicaciones en revistas nacionales e internacionales y autora de libros y capítulos de libro. barront@servidor.unam.mx

"The mission of the education for the planetary era is to fortify the

conditions of possibility of the emergency of a society-world com

posed by the protagonist citizens, conscious and critically

committed in the construction of a planetary civilization"

(Morín, 2003, P.122).

Among the discussed topics within the debates, it emphasizes the one of the educative systems and the necessity to review and transform them to face demands of a new nature, associated to a globalised world in which the societies are inserted.

PALABRAS CLAVE: Docente, formación, competencia, tutoría.

En la sociedad del conocimiento la importancia del capital centrado en el individuo radica en que es fuente de creación de ventajas, éstas proceden de información, formación, pericia, capacidad creativa, habilidad para identificar y resolver problemas, y liderar y gestionar convenientemente organizaciones productoras de satisfactores sociales. La institución educativa parece constituirse en el espacio privilegiado de creación del capital intelectual de la sociedad.

Se concibe a las instituciones de educación superior asociadas al progreso y a la transmisión del saber; sin embargo, en las últimas décadas cada vez es más frecuente que exista una desarticulación entre las necesidades del mercado de trabajo y la formación que ofrecen las instituciones, lo cuál se traduce en largas filas de desempleados o bien en un período cada vez mayor que transcurre para que estos ingresen a las fuentes de trabajo.

Esto hace necesario que se plantee una revisión profunda de los sistemas educativos para buscar alternativas de transformación que respondan al mundo globalizado que estamos viviendo. La educación, a pesar de las contradicciones y problemas que enfrenta, se considera como una opción viable para generar desarrollo sostenible en las diferentes sociedades. 
A nivel internacional y nacional existe una profunda preocupación por mejorar la calidad institucional mediante esfuerzos conjuntos y comprometidos. Como medidas apremiantes se propone el revisar y reordenar misiones, optar por rediseños innovadores que propongan nuevas formas de educación e investigación en el nivel superior.

La práctica docente no puede concebirse exclusivamente como una especialización o una práctica profesional y funcional en el ámbito educativo, requiere retomar su sentido político e incidir en el desarrollo de estrategias que permitan respetar y salvaguardar la vida humana, la diversidad cultural e ideológica, con la esperanza de construir una sociedad más humana.

Nuevos retos y articulaciones

El rol del profesor de educación superior se ha de transformar de transmisor del conocimiento, al de tutor o asesor del alumno, a fin de que alcance una formación que le prepare para un desenvolvimiento acorde con su proyecto de vida. En este sentido, la tutoría, como modalidad de la práctica docente, no suple a la docencia frente a grupo, sino que la complementa y la enriquece. Asimismo, puede ser vista como un instrumento de cambio, que podría reforzar los programas de apoyo integral de los estudiantes en el campo académico, cultural y de desarrollo humano, en la búsqueda del ideal de la atención individualizada de aquellos en su proceso formativo.

Con base en la perspectiva de los organismos internacionales como la Organización de las Naciones Unidas para la Educación, la Ciencia y la Cultura (UNESCO) y el Centro Regional para la Educación Superior en América Latina y el Caribe (CRESALC), quienes sugirieron la creación la figura del docente-tutor que apoyara el desarrollo integral del estudiante, así como también, de las políticas educativas nacionales, se generó la propuesta para que las instituciones educativas diseñaran un sistema institucional de tutorías.

La formación de los docentes obedece a las demandas de esta nueva sociedad globalizada y al compromiso de las Instituciones de Educación Superior (IES) por asumir una nueva visión y un nuevo paradigma para la formación de los estudiantes, basada en:

1) El aprendizaje a lo largo de toda la vida,

2) La orientación prioritaria hacia el aprendizaje autodirigido (aprender a aprender, aprender a emprender y aprender a ser) y

3) El diseño de nuevas modalidades educativas, en las cuales el alumno sea el actor central en el proceso formativo. 


\section{La calidad de la formación}

La calidad también se ha convertido en preocupación fundamental en el ámbito de la educación superior ya que la satisfacción de las necesidades de la sociedad y el cumplimiento de las funciones de este nivel educativo dependen, en última instancia, de la calidad del personal docente, de los estudiantes, de la infraestructura y, desde luego, de los programas educativos para la formación de profesionales. El interés por la calidad predomina en el debate sobre políticas de educación superior desde hace ya varios años, y no cabe duda que esta tendencia se mantendrá, dada su importancia para el desarrollo educativo y, aunque aún no hay acuerdo en torno a la forma de alcanzar la calidad, las instituciones de educación superior se encuentran hoy fuertemente comprometidas con este desafío. Gómez, Smith y Valle (1993: 25) plantean que la intencionalidad de la educación superior rebasa los propósitos de formación para el trabajo, así como las propuestas de una educación integral entendida como un ornamento cultural, por lo que sugieren una nueva definición de calidad profesional "es un proceso que asegura la adquisición de conocimientos significativos y el desarrollo de capacidades que permite al sujeto concebirse como inmerso en una realidad social de la que es parte activa y frente a la cual se desempeña no sólo como experto del conocimiento en una ámbito específico, sino como ciudadano competente".

No existe una sola acepción de calidad ni en el ámbito empresarial, de donde proviene su uso, ni en las instituciones educativas. En sus orígenes, se entendió la calidad en relación con los recursos humanos y materiales, y se establecieron proporciones entre bienes disponibles y recursos; más adelante el foco de la calidad se desplazó a los procesos (Glazman, 2001). En la actualidad el concepto de calidad está ligado tanto a la educación como al mercado, bajo el supuesto de que la educación debe ajustarse a las exigencias de la oferta y la demanda. En este sentido, se dice que la educación debe coincidir con las necesidades de la producción. En el fondo, el criterio de calidad promueve la estratificación entre las instituciones educativas; asimismo se constituye en un sinónimo de medición con referencia a normas estandarizadas y se llega a creer que existe un mejoramiento automático de las instituciones una vez que éstas han sido evaluadas.

La noción de calidad, en general, y su aplicación al campo educativo es un tema polémico. Para hablar de calidad en la educación superior se tiende a considerar aspectos tales como la eficacia y la eficiencia (Martínez Rizo, 1992). La eficiencia del sistema suele definirse como la relación entre los productos y los insumos que fueron necesarios para generarlos, es decir, como el costo de los productos (Martínez Rizo, 1992). Por su parte, el concepto de eficacia alude a la relación que se establece entre los productos y las necesidades del entorno; es la utilidad de 
los productos con respecto a las necesidades de la sociedad en la que está inmersa la institución. Los dos conceptos anteriores hacen referencia al contexto, a los insumos y al producto, sin tomar en cuenta los procesos, ni los actores del sistema. Ahora bien, al analizar la eficacia de las IES subyace siempre una visión sobre la educación, la cual puede ser vista como una inversión -en la medida que forma personal especializado para desempeñar determinadas funciones- o como un servicio -en la medida en que proporciona a sus egresados una formación general que se añade al bagaje mínimo de la educación básica (Martínez Rizo, 1992).

Competencias del docente

Los debates esenciales en torno a la formación de docentes, están estrechamente vinculados con los cambios culturales, políticos, sociales y económicos que están afectando a todas las sociedades.

La formación docente se entreteje con el desarrollo de los procesos de industrialización y los valores, saberes y prácticas se insertan en un contexto político y cultural específico.

En esta época el sentido de la formación docente se ha modificado; hay que recordar que el ideal formativo clásico que por años prevaleció en las instituciones escolares presuponía como fin último la formación humana. A la escuela se le asignaban las funciones de sustitución de la familia y propedéutica para el mundo del trabajo. En la actualidad, se enjuicia su calidad desde el pragmatismo, "no desde patrones cualitativos culturales o pedagógicos, según sus consecuencias sociales, sino mirando hacia el modelo del mercado como vía para estimular la eficiencia y eficacia de los sistemas escolares, incluido el sector público, apoyándose en las diferencias de la oferta para que los consumidores estimulen la competitividad" (Gimeno, 1995: 5).

En las dos últimas décadas la formación docente ha sido impactada por las exigencias y demandas de un mundo globalizado, señalando que todo docente debe cubrir como requerimientos básicos: el dominio de idiomas, el manejo de la informática (saber), el desarrollo de competencias interactivas, comunicacionales o socio-relacionales(saber ser), la temática de relaciones humanas, incluye la necesidad de manejo de personal, coordinación de grupos y trabajo en equipo. Los requerimientos denominados operativos(saber hacer), están vinculados a la dificultad de aplicar los conocimientos a situaciones concretas; finalmente la flexibilidad, la polivalencia y la versatilidad son cualificaciones que todo docente debe poseer. 
La competencia profesional del docente, entendida más como una competencia intelectual, trasciende el sentido puramente técnico del recurso didáctico. Las competencias docentes se caracterizan por ser complejas, combinan habilidades, principios y conciencia del sentido y de las consecuencias de las prácticas pedagógicas; así como una reflexión y análisis sobre los contextos que las condicionan y que van más allá del aula. La competencia profesional

"se refiere no sólo al capital de conocimientos disponibles, sino a los recursos intelectuales de que dispone con objeto de hacer posible la ampliación y desarrollo de ese conocimiento profesional, su flexibilidad y profundidad" (Contreras, 1999: 58).

La docencia universitaria aparece ligada a un conjunto de competencias didácticas en cuya génesis juega un importante papel el conocimiento teórico-práctico y la actividad reflexiva sobre la práctica.

En sentido amplio, se concibe a la competencia como un constructo molar que sirve para referirse a un conjunto de conocimientos y habilidades que los sujetos requieren para desarrollar algún tipo de actividad. Cada actividad exige un número variado de competencias que pueden ser desglosadas en unidades más específicas de competencia, en las que se especifican las tareas concretas que están incluidas en la competencia global. Se puede afirmar que la competencia está formada por diversas unidades de competencia.

Aubrun y Orifiamma (1990) clasifican las competencias en cuatro grandes grupos:

1:- Competencias referidas a comportamientos profesionales y sociales. Se refieren al tipo de actuaciones ordinarias que los sujetos han de realizar en la empresa en la que trabajen, tanto en lo que se refiere a actuaciones técnicas o de producción, como a las de gestión, a la toma de decisiones, al trabajo compartido, a la asunción de responsabilidades, etc.

2.-Competencias referidas a actitudes. Tiene que ver con la forma de afrontar la relación con las personas, las cosas, las situaciones que configuran el trabajo a desarrollar: la motivación personal, el compromiso, las formas de trato con los demás, la capacidad de adaptación, etc.

3.- Competencias referidas a capacidades creativas. Cómo los sujetos abordan el trabajo en su conjunto, si buscan soluciones nuevas, si asumen riesgos, si tratan de ser originales, etc. 
4.- Competencias de actitudes existenciales y éticas. Si el individuo es capaz de ver las consecuencias de las propias acciones profesionales, la capacidad para analizar críticamente el propio trabajo, si se pone un proyecto personal y fuerza para hacerlo realidad, si se posee un conjunto de valores humanísticos y de compromiso social y ético.

Existe un gran riesgo de confundir las actitudes de corte ético, con otras actitudes y valores ligados a procedimientos, los principios éticos no son meras normas lógicas $o$ técnicas metodológicas. Tampoco se pueden confundir los procedimientos con meras habilidades motrices, desvinculadas de conceptos y principios. La única posibilidad de enfrentar estos riesgos es la de profundizar las relaciones de los contenidos educativos con el conocimiento y con los valores.

En general, se puede afirmar que los retos que se presentan para la educación, así como también para los docentes y estudiantes, no sólo atañen al ámbito disciplinario y científico, sino también a una visión distinta de la realidad social y del conjunto de relaciones sociales en las que están insertos, En sentido amplio, la educación por definición es para la convivencia, aprender a vivir con otros, lo que implica saber vivir con otros y, este saber es enseñado por aquellos que tienen el poder de regular socialmente la convivencia (Cullén,1997).

Es importante enfatizar que la convivencia se construye cultural, histórica e ideológicamente, en el interjuego de las relaciones de poder. Enseñar a convivir es enseñar que las relaciones sociales deben basarse en la equidad y en la solidaridad. En este mundo globalizado los retos

para los docentes, emanados de una realidad dinámica, cambiante y contradictoria, se encaminan hacia el manejo de las incertidumbres y hacia la preparación para el riesgo, el azar, lo inesperado y lo imprevisto, dejando atrás una visión estática del mundo. Las certezas que en un momento dado se trabajaban en los distintos ámbitos disciplinarios, políticos y culturales, se han modificado; la esperanza de que la ciencia proporcione certezas ha quedado atrás, se esperaría que ayude a enfrentar racionalmente las incertidumbres.

Entender la educación como acción comunicativa implica que los participantes intervengan en un diálogo, donde cada uno busca comprender al otro y consensuar planes de acción, lo que permite una producción social de conocimientos con reglas claras de juego y con compromiso real de los participantes. En este sentido de acción comunicativa, la atmósfera educativa, es claramente ética. 
Zabalza (2003) señala las siguientes competencias didácticas del docente:

\section{1.-Competenciaplanificadora.}

Una competencia fundamental de los docentes la constituye la capacidad de planificar, desde el diseño del programa, la organización de los contenidos, la selección y organización de las estrategias de enseñanza, de aprendizaje y de evaluación.

Los programas de los grupos de los académicos, representan una interpretación por parte de los docentes de los programas oficiales-sintéticos, sobre todo cuando varios docentes imparten la misma materia. El programa del docente se construye a partir de los dos programas anteriores y, fundamentalmente, a partir de su experiencia profesional y de las condiciones particulares en las que trabaja. También es importante reconocer el proceso de negociación que realiza el docente a partir de su competencia profesional y las características e intereses de los alumnos. En este proceso de planificación se toman en cuenta las ideas pedagógicas de los docentes, los conocimientos disciplinarios y la experiencia didáctica (Zabalza, 2003).

2.- Competencia didáctica del tratamiento de los contenidos

En ésta, se pueden ubicar tres unidades de competencia: seleccionar, secuenciar y estructurar didácticamente los contenidos disciplinarios.

Seleccionar los contenidos a partir de los indicadores de vigencia, suficiencia /cobertura y relevancia, a partir de las siguientes preguntas, en cuanto al indicador de vigencia ¿los programas reflejan apropiadamente los avances y los enfoques actuales/vigentes de la disciplina?

Con relación al indicador de suficiencia/ cobertura se plantean los siguientes cuestionamientos: a) ¿los programas reflejan una visión amplia y plural de la disciplina? y b) tomando en cuenta el perfil profesional propuesto ¿qué aspectos de la formación profesional son los que se enfatizan en los programas elaborados?

En el indicador de congruencia se especifica si se encuentran algunos problemas de omisiones, repeticiones o sesgos en las unidades temáticas del programa.

En el indicador de relevancia se considera si los contenidos elegidos son relevantes para la formación profesional. (Barrón,2003). 
La secuenciación de los contenidos se refiere al orden en el cual se organizan los contenidos con la finalidad de propiciar aprendizajes significativos. Coll, Pozo, Sarabia y Valls (1992) reconocen desde un punto de vista constructivista tres tipos de contenidos: hechos y conceptos, procedimientos y actitudes. Dichos autores enfatizan el carácter diferenciado de la enseñanza, el aprendizaje y la evaluación de cada tipo de contenido.

La estructuración didáctica o presentación de los contenidos tiene que ver con la forma de comunicarlos, de explicarlos, de relacionarlos con la realidad y de cuestionarlos. Entrelazar las diversas indagaciones y observaciones, y articularlas entre sí hacia un fin determinado. De acuerdo con Merieu (2002) para la construcción del camino didáctico es necesario:

- Abrir caminos a través de la exploración; se requiere considerar no sólo el punto de llegada, sino el punto de partida.

- Dejar de privilegiar la dualidad objetivo-evaluación y añadirle objetivo-alumno, contenido-alumno, contenido método, método- evaluación.

- Establecer una relación pedagógica que incite al alumno al aprendizaje, que le despierte su deseo de saber.

- Considerar la brecha que se tendrá que abrir, no sólo en términos de objetivos sino de contenidos y condiciones para lograrla. Es decir, no sólo establecer lo que el alumno debe saber, sino lo que tiene que organizar y hacer tanto cognitiva, como afectivamente para lograrlo.

- Considerar las estrategias de enseñanza para provocar el aprendizaje.

- Crear situaciones movilizadoras, es decir de revisión y aplicación de conocimientos.

- Transformar los conceptos en acciones a realizar.

- Trazar un camino didáctico; inventariando las nociones y conocimientos previos necesarios para lograrlo, los procedimientos a seguir y los recursos que se utilizarán.

3:- Competencia comunicativa.

Esta competencia es inherente a rol del docente, en la que se enfatiza la capacidad para transmitir un mensaje con pasión a los alumnos e interesarlos por el conocimiento en general y por los asuntos concretos que desarrolla su disciplina. 
Como parte de la competencia comunicativa, el docente requiere manejar las nuevas tecnologías de la información y de la comunicación (internet, e-mail, multimedia, videoconferencia), lo que implica un cambio de roles. Se requiere de un docente que elabore guías de aprendizaje, y que tenga un contacto permanente con los educandos a través de la red. Su práctica se centrará más en ayudar y orientar al alumno para la selección y organización de la información, para la adquisición de habilidades, para el intercambio de información y de experiencias con sus pares nacionales y extranjeros. Quizás el gran reto sea el vivir en la incertidumbre en cuanto al manejo mismo de la información.

\section{4.-Competencia metodológica.}

Comprende todas aquellas acciones orientadas a gestionar la tarea docente, e implica la organización de los espacios que posibiliten ambientes de aprendizaje en los que los alumnos desarrollen un aprendizaje autónomo y significativo en escenarios reales de trabajo. El problema del método en el aula implica el crear condiciones para despertar $u$ orientar la curiosidad intelectual, establecer conexiones entre las diversas experiencias, formar hábitos, actitudes e intereses subyacentes y permanentes, interrelacionar la curiosidad orgánica de exploración física (tocar, manipular, palpar) y la interrogación lingüística (por qué, para qué, cómo).

"No existe una capacidad única y uniforme de pensamiento, sino una multitud de modos diferentes en los que las cosas específicas observadas, recordadas, oídas o acerca de las cuales se ha leído, evocan sugerencias o ideas pertenecientes a un problema o cuestión y que hacen avanzar la mente hacia una conclusión justificable. La formación consiste en este desarrollo de la curiosidad, la sugerencia y los hábitos de exploración y comprobación que aumenta la sensibilidad a los interrogantes y el amor a la investigación de lo desconcertante y desconocido, mejora la idoneidad de las sugerencias que se presentan a la mente, controla su sucesión en orden evolutivo y acumulativo, realiza el sentido de la fuerza, la capacidad de prueba de todo hecho observado y toda sugerencia recogida. El pensar no constituye un pensamiento mental aislado; por el contrario, es una cuestión relativa al modo en que se emplea la inmensa cantidad de objetos observados y sugeridos, el modo en que coinciden y en el que se les hace coincidir, el modo en que se los manipula. En consecuencia, ninguna asignatura, ningún tema, ninguna pregunta es intelectual por sí misma, sino por el papel que se le hace desempeñar en la dirección del pensamiento en la vida de toda persona (Dewey, 1989: 64). 
Todo lo que el docente realiza, así como el modo en que lo hace, incita al alumno a responder de una u otra forma, y cada respuesta tiende a dirigir la actitud del estudiante en uno $u$ otro sentido. Lo que implica diversificar las modalidades de trabajo en el aula a través de talleres, seminarios, laboratorios y aún más, a través de la posibilidad de promover la capacidad en el alumno de transferir el conocimiento del mundo del saber, la ciencia al mundo del trabajo profesional, y de establecer una relación compleja entre el saber y el trabajo en la esfera del conocimiento de alto nivel y de las tareas cognoscitivas complejas. Para el logro de este fin se proponen los internados y otras fases prácticas en los programas escolares, así como la participación de los profesionales en la enseñanza y en otras actividades académicas.

\section{5.-Competencia comunicativa y relacional.}

Se concibe como una competencia transversal debido a que las relaciones interpersonales constituyen un componente básico de las diversas competencias. La interacción docente alumno, en cualquier modalidad educativa es fundamental y dependiendo del rol que asuma el docente se generará una clima propicio o no para la participación, discusión y análisis en el aula.

6.- Competencia tutorial.

Una de las principales dificultades que surgen al intentar precisar el concepto de tutoría, se relaciona con la diversidad de significados en juego, ligados a un campo conceptual amplio y denso que remite a figuras distintas de la tutoría y por lo mismo de sus funciones y prácticas (Sánchez Puentes, 2000).

La tutoría también es concebida como una forma de atención educativa, donde el profesor apoya a un estudiante o a un grupo de estudiantes de una manera sistemática, por medio de la estructuración de objetivos, programas por áreas, técnicas de enseñanza apropiadas a la integración de grupos conforme a ciertos criterios y mecanismos de monitoreo y control, entre otros (Alcántara,1990). En la tutoría se propicia una relación pedagógica diferente a la que establece la docencia ante grupos numerosos. En este caso, el profesor asume el papel de un consejero o de un "compañero mayor"; el ejercicio de su autoridad se suaviza hasta casi desaparecer; el ambiente es mucho más relajado y amigable (Latapí, 1990). 
La Asociación Nacional de Universidades e Instituciones de Educación Superior (ANUIES, 2000) en su propuesta institucional de tutorías, la concibe como un proceso de acompañamiento durante la formación de estudiantes, que se concreta mediante la atención personalizada o a un grupo reducido de alumnos, por parte de académicos competentes y formados para esta función. Es distinta y a la vez complementaria a la docencia frente a grupo, pero no la sustituye.

En el discurso actual sobre tutorías, se ha generado un debate del sentido de acompañamiento que ésta implica, "la noción de acompañamiento parece haber emergido de las dificultades encontradas por los actores del campo educativo para responder a dos tipos de exigencia: la preocupación de un alumnado desorientado, considerado, no obstante, como ser autónomo o capaz de llegar a serlo, y la exhortación de resultados, de excelencia y de eficacia, siempre más altos, provenientes de los responsables de la institución" Ghouali (2007: 208).

Desde esta posición, acompañar se define como un proceso que dinamiza tres lógicas: relacional, espacial y temporal. La relacional concebida como una conexión, unirse a alguien; la espacial referida a un desplazamiento, para ir a donde él va, y la temporal referida a estar con el otro al mismo tiempo (Ghouli, 2007).

Para Ardoino (2000) sólo se puede acompañar a alguien si esta persona sabe a dónde va. El acompañante puede existir como persona, pero reconoce que el acompañado es él, el sujeto de la acción. En este proceso se genera una relación de intersubjetividad "en donde los sujetos se comunican en dos posiciones diferentes, donde el acompañante se pone en juego y acepta que el acompañado esté con él en una relación de alteración mutua. Donde el otro cambia al mismo tiempo que intenta cambiarlo. A través de esta idea, la dimensión ética del acompañado es subyacente" (Ardoino,2000: 7-8).

La tutoría puede estar ligada al apoyo que el docente proporciona al alumno en su trayectoria escolar, para la toma de decisiones acertadas, recomendándole bibliografía o sólo intercambiando opiniones sobre la vida académica de la institución; también puede ubicarse en relación con la formación de investigadores en un campo científico en particular. Cuando se habla de la tutoría para enseñar a investigar, se refiere a funciones distintas, con otro perfil del tutor y con condiciones institucionales particulares apropiadas al logro de este propósito. Lo que aquí hace la diferencia no es propiamente la naturaleza de la investigación, sino el uso que se le da a la misma, y en particular, las condiciones institucionales que la acompañan. 
En ambas prácticas se requiere del compromiso de ambos actores, del estudiante y del tutor insertos en un espacio académico, en donde se establecen lazos particulares a través de las relaciones diarias, vínculos, delimitación de identidades, expectativas, ritos, mitos y creencias, etc. La vida académica se expresa través de lo micro, de los detalles triviales, de la comunicación diaria. Esta última tiene como ámbito lo intelectual, pero también lo afectivo.

En el ámbito de la investigación, la figura del tutor alude al del investigador que se responsabiliza académicamente del estudiante de manera inmediata directa y permanente; establecen una relación de trabajo y crean un proyecto de formación específico para el estudiante (Sánchez, 2000). Es, en este espacio mediado por múltiples situaciones, en donde se pone en juego la formación del futuro investigador, "la formación es un proceso de transmisión y asimilación de saberes relacionados con una disciplina, de las habilidades particulares de ésta y de una concepción sobre la vida. Ésta es posible si la persona tiene compromiso con su formación, si está dispuesta a cultivar constantemente su disciplina" (Piña,2000: 89).

Lo más relevante en este proceso es el hecho de que el tutor transmite fundamentalmente un oficio, el de investigador. Sánchez Puentes (2000) señala que el investigador tutor transmite tres tipos de saberes: los teóricos, los prácticos y los significativos.

a) Los saberes teóricos se refieren a las teorías y conocimientos reconocidos socialmente, paradigmas, supuestos teóricos y marcos conceptuales.

b) Los saberes prácticos, conforman el conjunto de estrategias, habilidades, destrezas propias del oficio del investigador. A través de este tipo de saberes se enseña y se aprende a problematizar, a fundamentar; a concebir la teoría como una herramienta de análisis; a organizar, analizar e interpretar la información; cada uno de estos quehaceres de la investigación está integrado por numerosas y diferentes operaciones que requieren conocimientos teóricos, habilidades y estrategias.

c) Los saberes significativos, el tutor también enseña a cuestionar acerca de los fines últimos de la investigación, los por qué de carácter ético-político del saber científico, así como los para quiénes de la producción científica. Se refiere a los significados y objetivos últimos del conocimiento que se genera.

Estos tres tipos de saberes insertos en las tradiciones disciplinarias, en procesos de socialización y circulación, y en las posibilidades institucionales reales permean la formación de las nuevas generaciones de profesionales. Una generación es más que un grupo humano coincidente en un espacio y en un tiempo históricos, es 
antes que nada, heredera y por lo tanto receptora de múltiples influencias; pero a su vez estas condiciones de recepción le abren la posibilidad de consolidar, en los ámbitos institucionales, la producción, y en ella, no sólo están insertas las visiones que se plantearon en su formación, sino que cobran expresión en las orientaciones que guían al intelectual dentro de las premisas de los que se convertirán en los líderes de las nuevas generaciones.

La utilización de modelos centrados en el alumno y la orientación hacia el aprendizaje por la vía de la tutoría académica, requiere de la capacitación y la colaboración por parte de los distintos actores universitarios.

En la práctica los docentes-tutores articulan diversos saberes procedentes de su formación profesional, disciplinaria, curricular, experiencial o práctica, construidos a lo largo de su vida y de su trayectoria profesional; en este sentido Tardif (2004) señala la importancia de reconocer el entramado de relaciones desde las cuales construyen las prácticas los docentes-tutores: los saberes, el tiempo y el trabajo. En gran medida lo que saben los docentes sobre la enseñanza, proviene de su experiencia como alumnos y a partir de ésta construyen y reconstruyen sus prácticas en el aula, a través de un permanente movimiento de continuidad y rupturas con las teorías y perspectivas teóricas desde las cuales cobra sentido. Lo que implica el reconocimiento de que los saberes que fundamentan la enseñanza son existenciales, sociales y pragmáticos. Son existenciales porque involucran a los seres humanos en su totalidad, con sus anhelos, sus deseos, sus emociones, sus relaciones con los otros y consigo mismo; sociales, provenientes de distintos núcleos y tiempos de formación escolar y de la vida cotidiana; y son, por último, pragmáticos porque aluden a las experiencias y prácticas en el marco de las instituciones escolares y de las prácticas profesionales del docente.

Los saberes profesionales del docente-tutor pueden ser agrupados en: temporales, plurales y heterogéneos, personalizados y situados, y llevan consigo las señales de su objeto, que es el ser humano (Tardif, 2004).

Son temporales en tres sentidos: porque provienen de su historia de vida personal y escolar, de ritos iniciáticos y de rutinas que le dan seguridad en el aula, y de las prácticas escolares institucionalizadas (Zeichner y Gore,1990); (Carter y Doyle,1996). Son plurales y heterogéneos en función de las diversas situaciones a las que se enfrentan cotidianamente (Doyle,1986). Son personalizados y situados, porque se trata de saberes apropiados, incorporados, subjetivados; saberes que no son posibles disociar de la persona, de su experiencia y de su situación de trabajo. 
Con base en todo lo anterior, se puede afirmar que para transformar la educación se requiere que el docente-tutor realice una revisión a fondo de las relaciones con el conocimiento, a partir de la reflexión y del análisis del reconocimiento de algunos resabios en su prácticas, tales como los resabios emanados del "platonimo", es decir de un mundo de ideas fijado de una vez y para siempre, de la negación de su historicidad, su condicionamiento social y político, y de los resabios del iluminismo; se trabajan sólo con certezas, verdades inmutables y con un autoritarismo dogmático.

La utilización de modelos centrados en el alumno y la orientación hacia el aprendizaje por la vía de la tutoría académica, requiere de una formación específica y de la colaboración por parte de los distintos actores universitarios.

Los retos que se presentan para la formación del docente-tutor no sólo atañen al ámbito disciplinario y científico, sino también a una visión distinta de la realidad social y del conjunto de relaciones sociales en las que se inserta el profesionista, entre los que se pueden destacar los siguientes: la incertidumbre, la ética de la comprensión, la pertinencia del conocimiento y la comprensión (Morín, 1999).

Con relación a la incertidumbre, se puede señalar que las certezas que en un momento dado se trabajaban en distintos ámbitos, en lo económico, en lo político y en lo cultural, se han modificado; la realidad es dinámica, cambiante y contradictoria, y hoy más que nunca, la formación docente ha de encaminarse hacia el manejo de las incertidumbres, es decir, prepararse y preparar para el riesgo, el azar, lo inesperado y lo imprevisto, dejando atrás una visión estática del mundo.

La ética de la comprensión requiere de comprender la incomprensión, de argumentar y refutar, la comprensión no acusa, ni excusa, con ello se intentaría aprehender en conjunto el texto y el contexto, el ser y su entorno, lo local y lo global, lo multidimensional, en resumen lo complejo, es decir las condiciones del comportamiento humano. (Morín, 1999: 55).

La pertinencia del conocimiento implica reconocer el contexto en el que se genera y desarrollan los conocimientos, tener una visión holística de la realidad reconociendo la interrelación entre todos los elementos que la componen y sus múltiples dimensiones. Por consiguiente, tomar en cuenta todos estos aspectos, lo que implica reconocer la complejidad de los conocimientos que se pretenden manejar.

Formarse para la comprensión implica dos niveles, uno que atañe a la comprensión intelectual u objetiva y el otro, a la comprensión humana intersubjetiva. (Morín,1999: 51). Ambos, son necesarios para la convivencia en el 
mundo actual, en el que paradójicamente, los procesos de globalización han traído consigo procesos de exclusión, y con ello el resurgimiento de las xenofobias, los fundamentalismos y el racismo, aunado a las exigencias de competitividad a ultranza, emanadas de las reglas del libre mercado.

Finalmente, se puede afirmar que los nuevos perfiles generados por las exigencias de un mundo globalizado, requieren a su vez de una reorientación y de actualizar contenidos, metodologías y formas de trabajo en el aula. Como parte imprescindible de esta formación, la UNESCO señala que la educación superior del futuro deberá integrar valores como la solidaridad, la mística del trabajo humano, la responsabilidad, los derechos humanos, el respeto a la paz y al entorno y la consolidación de la identidad cultural y social (Pallán, 1997).

Los cambios académico-administrativos deberán prestar más atención a la intención de los docentes.

Fullan y Hargreaves (1999) señalan al respecto, es necesario:

- Dar voz a las intenciones del docente-tutor,

- Escuchar activamente la voz del docente-tutor,

- Crear oportunidades para que los tutores enfrenten las suposiciones y creencias que fundamentan sus prácticas, mostrar disposición a escucharles acerca del cambio,

- Evitar crear una cultura de dependencia por sobreestimar la veracidad de las investigaciones publicadas y subestimar el conocimiento práctico de los tutores,

- Evitar las modas en la forma de una implementación uniforme de nuevas estrategias sin cuestionarlas,

- Facultar a los docentes-tutores y sus escuelas para recuperar una responsabilidad sustancial en la toma de decisiones importantes, crear una comunidad de docentes-tutores que discuta y desarrolle sus intenciones en conjunto.

Existen diversos factores que se deben de considerar en la formación de los docentes-tutores:

- Los períodos en los cuales los docentes se educan e ingresan a la profesión,

- Los sistemas de valores y las tendencias educativas dominantes que coinciden con esos períodos, 
- La etapa de la vida y de la carrera en que se encuentra el docente-tutor,

- El efecto que tiene sobre su confianza en su propia capacidad educativa, su sentido de realismo y sus actitudes hacia el cambio.

- El género es otro factor importante que hay que tomar en cuenta.

El contexto de la tutoría:

- Para los docentes-tutores el cambio no es simplemente rehacer un diagrama de flujo, es algo que debe ocurrir en el mundo complejo y atareado de su aula.

- Es necesario saber el contexto donde el docente-tutor trabaja; como influye el ambiente en la tutoría, se requiere una comprensión ecológica de su actividad, el modo en que éste se desarrolla para adecuarse al medio, y en qué podemos y debemos cambiar ese medio si pretendemos modificar lo que en él ocurre.

- La tutoría no siempre es igual, no se puede estandarizar.

- Un aspecto importante de la tutoría es su realismo y practicidad. A la mayoría de los tutores les interesa la excelencia en el aula, la motivación de sus alumnos, hacer del aprendizaje un proceso activo y divertido. Pero también les interesa conservar su salud y evitar el estrés.

- Las características contextuales fijan límites muy definidos a lo que pueden hacer los tutores y a las posibilidades reales de innovación. Muchas de estas realidades, como el aislamiento en el aula, están firmemente institucionalizadas y tienen profundas raíces históricas. Algunas de estas realidades son: el tamaño del grupo, el tiempo, y el currículo.

Las acciones emprendidas hasta la fecha reflejan una gran preocupación y movimiento al interior de la mayoría de las instituciones de educación superior, cada una de acuerdo a sus recursos, a la cultura organizacional que le identifica y a las intenciones de quienes las dirigen, que muestran como están tratando de incorporarse a esta nueva visión de hombre que se requiere formar en un mundo globalizado; indudablemente los recursos humanos que se han movido para enfrentar el reto de la formación de tutores son muy variados, lo que se puede rescatar como un denominador común es el deseo de conocer a qué responde este nuevo rol y cuáles son las funciones que se tienen que desempeñar. Coraggio y Torres (1997) señalan que sin docentes de calidad no es posible una educación escolar de calidad. La verdadera reforma educativa, sobre todo en el ámbito curricular y pedagógico, que es el que finalmente importa, se juega en el terreno docente. 
El rol docente sólo puede modificarse en el marco de una profunda transformación del orden escolar. Cambiar el rol y el profesionalismo docente sólo puede hacerse como parte de un cambio sistémico que compromete a la institución escolar, así como al modelo de pensar y hacer política educativa tanto a nivel nacional como internacional.

Fuentes de Consulta

ALCÁNTARA, Armando. Consideraciones sobre la Tutoría en la Enseñanza Universitaria. Perfiles Educativos, México: CISE-UNAM, n. 49-50, p. 51-55. 1990.

ANUIES. Programas institucionales de tutoría. Una propuesta de la ANUIES para su organización y funcionamiento en las instituciones de educación superior. México, ANUIES, 2000, 163p.

AUBRUN, S. y ORIFIAMMA, R. Les competences de 3em.dimensión. París Consevatorio de arts e Metiers, 1990.

BARRÓN, C., GARDUÑO, G. y GUTIÉRREZ, N. La teoría de las generaciones como una categoría analítica de estudio en la formación del profesional universitario. En: DÍAZ BARRIGA Ángel. (coord.) Currículo, evaluación y planeación educativa. México, COMIE, 1995, p. 119-132

BARRÓN, C. Análisis de los contenidos curriculares. Un acercamiento metodológico. En: CHEHAYBAR Y KURI, Edith y AMADOR, Rocío Amador (Coord). Procesos y prácticas de la formación universitaria. Pensamiento Universitario. México, CESU-UNAM, n. 93, p. 75-96, 2003.

BARRÓN, Concepción y GUTIERREZ, Norma. La formation des tuteurs pour la recherche en sciences sociales dans le mexique d'aujourd'hui: analyse des strategies. En: CONGRES DE L'AFIRSE, Pau, Francia, 2002. p.1-15

BARRÓN, Concepción. La docencia y la tutoría: nuevos retos, nuevas articulaciones. VI FORO DEL CEU, LA UNIVERSIDAD: ENTRE LO PRESENCIAL Y LO VIRTUAL. México: Universidad Autónoma del Estado de México, 2003.

CORAGGIO, José Luis y TORRES, Rosa Maria. La educación según el Banco Mundial. Un análisis de sus propuestas y métodos. Buenos Aires: Miño y Dávila CEM, 1997.

COLL, Cesar, POZO, Juan Ignacio, SARABIA, Bernabé. y VALLS, Enric. Los contenidos de la reforma. Enseñanza y aprendizaje de conceptos, procedimientos $y$ actitudes, Madrid : Aula xxi: Santillana, 1992, 202p.

CONTRERAS, José. La autonomía del profesorado. Madrid: Morata, 1999, 231p. 
CULLÉN, Carlos. Crítica de las razones de educar. Temas de filosofía de la educación. Buenos Aires. 1997, 60 p.

DÍAZ BARRIGA, Ángel. Tarea docente. Una perspectiva didáctica y psicosocial. México: Nueva Imagen, 1993. p. 39-99.

DEWEY, John. Cómo pensamos. Nueva exposición de la relación entre pensamiento reflexivo y proceso educativo. España: Paidós. 1989, 249p.

CARTER, K. y DOYLE, W. Personal narrative and life history in leraning to Teach. En: SIKULA,J.,BUTTERY,T.J. \& GUYTON, E (dir).Hanbook of

Research on Teacher Education. 2nd ed. New York: Macmilan, 1996, p.120 142.

DOYLE,W. Classroom organization and management. En: WITTROCK, M.C.(dir). Handbook of Research on teacher education. New YorK: Macmillan, 1986, p392431.

GLAZMAN, Raquel. Evaluación y exclusión en la enseñanza universitaria. México. Paidos. 2001, 181p.

GIMENO, José. "Paradigmas crítico-reflexivos en la formación de profesores. Derivaciones para los contenidos y para las prácticas". Ponencia presentada en EL SIMPOSIO INTERNACIONAL FORMACIÓN DOCENTE, MODERNIZACIÓN EDUCATIVA Y GLOBALIZACIÓN. México: UPN. 1995, 1-23 p.

GÓMEZ, José, Smith Marcia y VALLE Ángeles. Formación Profesional y calidad de la educación. Perfiles Educativos, México, n. 47-48. ene-jun. 1993, p. 24-32.

FULLAN, Michael y HARGREAVES, Andy. La escuela que queremos. Amorrortu/SEP, Biblioteca para la Actualización del Maestro. México, 1999.

LATAPÍ Sarre, P., La enseñanza tutorial: Elementos para una propuesta orientada a elevar la calidad. En: Revista de la Educación Superior, n. 68, octubre-diciembre, México: ANUIES, 1988, p. 5-19.

MORIN, Edgar. Los sietes saberes necesarios para la educación del futuro. México : UNESCO : Correo de la UNESCO. 1998. 107.p.

MARTÍNEZ RIZO, Felipe. La calidad de las instituciones de educación superior. Su evaluación y su promoción. Cuadernos de Planeación Universitaria. $3^{\mathrm{a}}$ época. Año 6, n. 2. México. UNAM. 1992, 143 p.

MERIEU, Phillipe. Aprender sí ¿pero como?. Barcelona: Octaedro, 2002. 
MORÍN, Edgar, ROGER E., MOTTA, Raúl. Educar en la era planetaria. España: Gedisa, 2003.

MORA, José Antonio. Acción tutorial y Orientación Educativa. España: Narcea, 1998.

PALLÁN, Carlos. Impacto de la innovación en la organización académica. En: Innovación curricular en las instituciones de educación superior. México.

ANUIES. 1997. p.21-31.

SÁNCHEZ PUENTES, R. y SANTA MARÍA M. El proceso y las prácticas de tutoría. En: SÁNCHEZ PUENTES, Ricardo y ARREDONDO, Martiniano. (coord.). Posgrado en Ciencias Sociales y humanidades. Vida académica y eficiencia terminal. México: CESU-UNAM, 2000, p.109-136.

PIÑA OSORIO, J. M. y Mireles, O. El proceso de socialidad y de vida académica. En: SÁNCHEZ PUENTES, Ricardo y ARREDONDO, Martiniano (coord..) Posgrado en Ciencias Sociales y Humanidades. Vida académica y eficiencia terminal. México: CESU-UNAM, 2000, p.79-108.

TARDIFF, Maurice. Los saberes del docente y su desarrollo profesional. Madrid. Narcea, 2004, 233p.

SCHÖN, Donald. La formación del profesional reflexivo. Barcelona .México: Paidos. 1998, p. 330.

ZABALZA, Miguel A. Competencias docentes del profesorado universitario. Calidad y desarrollo profesional. Madrid: Narcea, 2003.

ZEICHNER, KENNETH y GORE, Teacher socialisocialization. En: Houston, R.W. (ed.) Handbook of Research on Teacher Education. Nueva York. Macmillan, 1990, p. 329-348. 